\title{
Dependence of Dendritic Side-branches on Parameters in Phase-Field Simulations
}

\author{
Zhu Changsheng ${ }^{1,2}$, Liu Baicheng ${ }^{3}$, Jing Tao $^{3}$ and Feng Wenfang ${ }^{1}$ \\ ${ }^{1}$ Lanzhou University of Technology, Lanzhou 730050, P. R. China \\ ${ }^{2}$ State Key Laboratory of Gansu New Non-Ferrous Metal Materials, Lanzhou 730050, P. R. China \\ ${ }^{3}$ Department of Mechanical Engineering, Tsinghua University, Beijing 100084, P. R. China
}

The influence of phase-field parameters on dendritic side-branches is studied by using a phase-field model coupled with thermal noise. The result of calculation indicates that, the thermal diffusion layer collected around the dendrite tends to become thinner with the undercooling increasing, which is favorable to the growth of the side-branches and the dendrite takes on the morphology of developed side-branches; The less coupling coefficient $\lambda$ is, the quicker the dendrite tip velocity converges on the Green function calculation, the more developed the side-branches are; The anisotropy coefficient $\varepsilon$ influences the steady state of the dendrite tip, the largeris, the greater the dendrite tip velocity is, the more developed the side-branches are and the smaller the DAS is; The magnitude of thermal noise $F_{\mathrm{u}}$ has an obvious effect upon temperature field, when appropriate value is assigned to $F_{\mathrm{u}}$, the noise can promote the emergence of side-branches, but it does not influence the tip operating state.

(Received July 22, 2004; Accepted November 1, 2004)

Keywords: phase-field, dendrite, thermal noise, side branches, undercooling

\section{Introduction}

Dendrites are intricate patterns that make up the microstructure of many important commercial alloys. They develop a complex shape due to the emission of secondary branches behind the growing tips of primary branches. ${ }^{1)}$ The side-branching mechanism requires some continuous source of noise at the tip. Therefore, thermal noise, originating from microscopic scale fluctuations inherent in bulk matter, is the most natural and quantifiable candidate to be considered. ${ }^{1)}$ Barber et $a .^{2)}$ studied the evolution of time-dependent deformations of the needle crystal (Ivantsov) solution of the two-dimensional symmetric model of solidification in the limit of small Péclet number within a WKB approximation. The amplitude of a localized wave packet grows exponentially as $z^{1 / 4}$, where $z$ is measured from the tip along the symmetry axis of the dendrite. Brener and Temkin ${ }^{3)}$ considered anisotropic needle crystals in three dimensions and concluded that experimentally observed side-branches could be explained by considering noise of a thermal origin. The growth of the side-branches amplitude was found to behave exponentially as a function of $z^{2 / 5}$, which is faster than $z^{1 / 4}$ dependence obtained in the axisymmetric case. ${ }^{4)}$ The side-branches wavelength was found to be as a function of $z^{1 / 5}$, very similar to that obtained in the axisymmetric case. Recently, few studies of side-branches with a phase-field model have been carried out, ${ }^{4-9}$ ) they obtained good quantitative agreement between the computed side-branches amplitude as a function of distance to the tip and the prediction of the linear WKB theory for anisotropic crystals in two dimensions. ${ }^{1,5)}$ In this paper, dendritic side-branches during crystal growth is investigated by simulation of a phase-field model which incorporates thermal noise of microscopic origin.

\section{Mathematical Model and Numerical Issues}

Recently, methods to incorporate thermal noise in the phase-field model have been developed by Karma and Rappel. ${ }^{1)}$ Although some previous phase-field simulations have obtained dendritic side-branches that resemble those observed in experiments, ${ }^{6-8)}$ those side-branches were generated by either numerical noise or randomly driving the tip. In the model of Karma and Rapple, thermal noise was incorporated in a thermodynamically consistent manner using the Langevid formalism. The nonconserved noise and conserved noise were added to the phase equation and the energy conservation equation respectively in the following manner:

$$
\begin{aligned}
\tau(\vec{n}) \partial_{t} \phi= & {\left[\phi-\lambda \mu\left(1-\phi^{2}\right)\right]\left(1-\phi^{2}\right)+\nabla\left[W^{2}(\vec{n}) \nabla \phi\right] } \\
& +\sum_{m=x, y} \partial_{m}\left[|\nabla \phi|^{2} W(\vec{n}) \frac{\partial W(\vec{n})}{\partial\left(\partial_{x} \phi\right)}\right]+\theta(\vec{r}, t) \\
\frac{\partial u}{\partial t}= & D \nabla^{2} u+\frac{1}{2} \frac{\partial p(\phi)}{\partial t}-\nabla \cdot \vec{q}(\vec{r}, t)
\end{aligned}
$$

The dimensionless temperature $u$ is defined as $\left(T-T_{\mathrm{M}}\right) /$ $\left(L / C_{\mathrm{p}}\right)$, where $T_{\mathrm{m}}, L$ and $C_{\mathrm{p}}$ are the melting temperature, latent heat and specific heat at constant pressure, respectively. $D$ is the thermal diffusivity, $\lambda$ is coupling constant, both $\tau$ and $W$ are functions of the interfacial normal $n$ in order to account for anisotropic surface energy and kinetics, respectively:

$$
\begin{aligned}
& \tau(\vec{n})=\tau_{0}\left[1+\frac{4 \varepsilon_{4}}{1-3 \varepsilon_{4}} \frac{\left(\partial_{x} \phi\right)^{4}+\left(\partial_{y} \phi\right)^{4}}{|\nabla \phi|^{4}}\right] \\
& W(\vec{n})=W_{0}\left(1-3 \varepsilon_{4}\right)\left[1+\frac{4 \varepsilon_{4}}{1-3 \varepsilon_{4}} \frac{\left(\partial_{x} \phi\right)^{4}+\left(\partial_{y} \phi\right)^{4}}{|\nabla \phi|^{4}}\right]
\end{aligned}
$$

$\vec{\theta}$ and $\vec{q}$ stand for the thermal noise vector, obeying Gaussian distribution with variances respectively:

$$
\begin{aligned}
\left\langle\theta(\vec{r}, t) \theta\left(\vec{r}^{\prime}, t\right)\right\rangle & =2 F_{\phi} \delta_{m n} \delta\left(\vec{r}-\vec{r}^{\prime}\right) \delta\left(t-t^{\prime}\right) \\
\left\langle q_{m}(\vec{r}, t) q_{n}\left(\overrightarrow{r^{\prime}}, t\right)\right\rangle & =2 D k_{\mathrm{B}} \frac{C_{\mathrm{p}} T_{\mathrm{M}}^{2}}{L^{2}} \delta_{m n} \delta\left(\vec{r}-\vec{r}^{\prime}\right) \delta\left(t-t^{\prime}\right)
\end{aligned}
$$


where $k_{\mathrm{B}}$ is the Boltzmann constant and $\delta$ is the delta function. Using $W_{0}$ as a length scale and $\tau_{0}$ as a time scale, all dimensional variables are cast into their dimensionless form as $\vec{r} / W_{0} \rightarrow \vec{r}, t / \tau \rightarrow t, D \tau_{0} / W_{0}^{2} \rightarrow D, \tau \theta \rightarrow \theta, \vec{q} \tau_{0} / W_{0} \rightarrow \vec{q}$. Then, the dimensionless variances of the thermal noise vector are given by:

$$
\begin{aligned}
\left\langle\theta(\vec{r}, t) \theta\left(\vec{r}^{\prime}, t\right)\right\rangle & =2 F_{\phi} \delta_{m n} \delta\left(\vec{r}-\overrightarrow{r^{\prime}}\right) \delta\left(t-t^{\prime}\right) \\
\left\langle q_{m}(\vec{r}, t) q_{n}\left(\vec{r}^{\prime}, t\right)\right\rangle & =2 D F_{\mathrm{u}} \delta_{m n} \delta\left(\vec{r}-\overrightarrow{r^{\prime}}\right) \delta\left(t-t^{\prime}\right)
\end{aligned}
$$

$F_{\mathrm{u}}$ is the magnitude of the thermal noise and is defined as

$$
F_{\mathrm{u}}=\frac{k_{\mathrm{B}} T_{\mathrm{M}}^{2} C_{\mathrm{p}}}{L^{2} W_{0}^{2}}=\frac{k_{\mathrm{B}} T_{\mathrm{M}}^{2} C_{\mathrm{p}}}{L^{2} d_{0}^{2}}\left(\frac{d_{0}}{W_{0}}\right)^{2}=F_{\exp }\left(\frac{d_{0}}{W_{0}}\right)^{2}
$$

The phase field eq. (1) is solved by using the Euler algorithm, while ADI algorithm is applied to the heat eq. (2). The space step is selected as $\Delta x \leq W_{0}$, the time step is specified by this way that $\Delta t \leq \frac{\tau_{0} \Delta x^{2}}{5 W^{2}}$ for keeping stable under the time-step iterations. In order to save the computational costs, the computation region is extended according to the increasing dendritic scale.

For an initial nucleus of the radius $r_{0}$,

$$
\begin{array}{lll}
\text { When } & x^{2}+y^{2} \leq r^{2}, & \phi=1, u=0 \\
\text { When } & x^{2}+y^{2}>r^{2}, & \phi=-1, u=-\Delta
\end{array}
$$

Where $x$ and $y$ are the coordinate axes, $u$ is dimensionless temperature.

The Zero-Neumann boundary conditions for $\phi$ and $u$ are imposed on the boundaries of the computational domain.

\section{Numerical Results and Discussion}

\subsection{The effect of undercooling on side-branches}

The undercooling has great effects on both dendritic nucleation and growth. The lower the undercooling is, the smaller the nucleation rate is, in other words, in the same volume of melt, each nucleus possesses more free space to grow. On the other hand, with the decrease of undercooling, the growth velocity slows down and the dendrite needs more time to grow. The simulation was performed by using relevant computational and material parameters as follows: $d_{0} / W_{0}=0.277, \tau_{0}=1, \Delta x=\Delta y=0.8, \Delta t=0.06, \varepsilon=$ $0.05, F_{\mathrm{u}}=2 \times 10^{-4}, \Delta=0.65$, Fig. 1(a) shows the equiaxed dendrite without side-branches obtained by simulation dimensionless temperature $\Delta=0.2$ and growth time $t=650,000 \Delta t$, Fig. 1 (c) presents the equiaxed dendrite with developed side-branches under high dimensionless undercooling oof $\Delta=0.65$ and growth time $t=26,000 \Delta t$. The corresponding temperature distribution results near equiaxed dendrite (illustrated in Fig. 1(a), Fig. 1(c)) are presented in Fig. 1(b) and Fig. 1(d) respectively. From the figures, it can be found that under the low undercooling, the thermal diffusion layer surrounding equiaxed dendrite is thicker than that under the high undercooling. During the growing of equiaxed dendrite, the latent heat releases to the melt through the thermal diffusion layer, the thermal diffusion thickness is $D_{T} / V_{\mathrm{c}}$. The growth velocity under low undercooling is slower than that under high undercooling, so the thermal diffusion length under low undercooling melt is thicker than that under high undercooling melt, which causes equiaxed

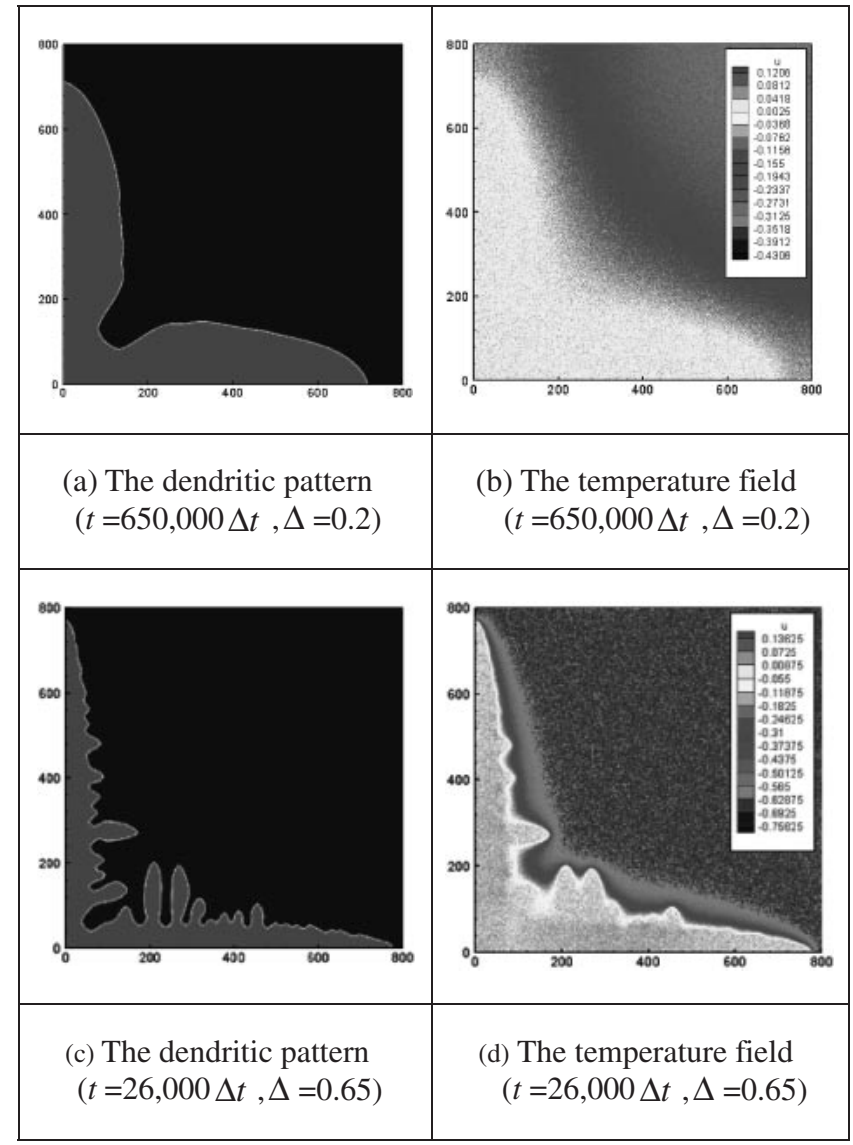

Fig. 1 The effect of undercooling on side-branches.

dendrites surrounded by a thicker thermal diffusion layer. The thermal diffusion layer baffles the release of latent heat and restricts the perturbation of the interface, as a result, sidebranches are suppressed, so equiaxed dendrites take on the morphology of no side-branches. Moreover, under high undercooling, the thin thermal diffusion layer avails the release of latent heat and promotes the development of the side-branches, therefore, the equiaxed dendrite shows a morphology of highly developed side-branches.

\subsection{The effect of coupling coefficient on side-braches}

The coupling coefficient $\lambda$ of the temperature field and the phase field is a very important parameter to represent the relation between energy and solidification, and determine the dendrite growth pattern. The coupling coefficient $\lambda$ should be set according to the physical parameters, but the ideal value should be obtained by experimentation. However, the test for it has not be found yet. Certain relation between $\lambda$ and dendrite tip velocity was showed in Fig. 2, when the coupling coefficient is less, the dendrite tip velocity converges quickly on the calculated value of the Green function (expressed with the dotted line). As the increment of coupling coefficient $\lambda$ continuously, the dendrite tip velocity reduces rapidly, thus, the stable state behavior of the dendrite tip is influenced. Karma et $a l .{ }^{1)}$ studied the convergence of dimensionless tip velocity as a function of $W_{0} / d_{0}$. In terms of the expression $d_{0}=a_{1} \frac{W_{0}}{\lambda},{ }^{11)}$ where $a_{1}$ is a positive constant of order unity, $a_{1}=I / J, I=2 \sqrt{2} / 3, J=16 / 15, F_{\mathrm{u}}=2 \times 10^{-4}$, therefore, 


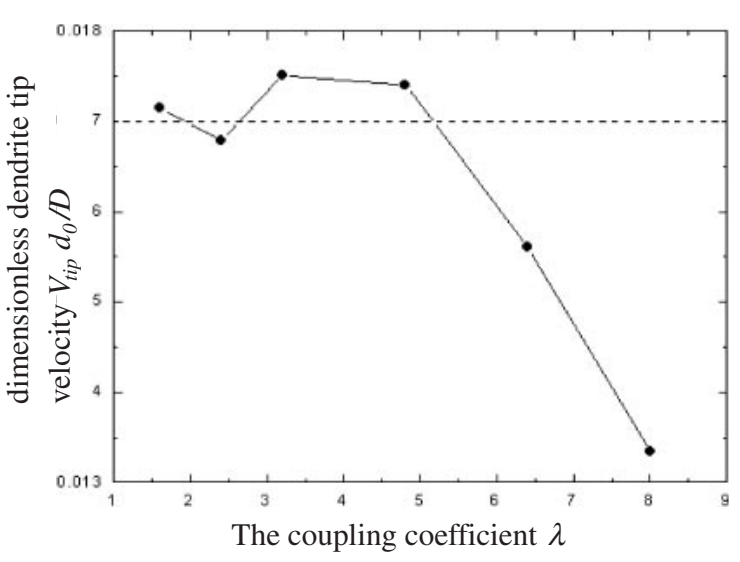

Fig. 2 The effects of the coupling coefficient on tip velocity.

Table 1 The relation of $d_{0} / W_{0}, W_{0} / d_{0}$ and $\lambda$.

\begin{tabular}{cllllll}
\hline$d_{0} / W_{0}$ & 0.555 & 0.416 & 0.277 & 0.185 & 0.139 & 0.111 \\
\hline$W_{0} / d_{0}$ & 1.8 & 2.4 & 3.6 & 5.4 & 7.2 & 9.0 \\
\hline$\lambda$ & 1.6 & 2.1 & 3.2 & 4.8 & 6.4 & 8.0 \\
\hline
\end{tabular}

the coupling coefficient $\lambda$ can be expressed as $\lambda=a_{1} \frac{W_{0}}{d_{0}}$. It can be seen the relation of $d_{0} / W_{0}, W_{0} / d_{0}$, and $\lambda$ by the data as shown in Table 1 . The simulation was performed by using relevant computational and material parameters as follows: $\Delta x=\Delta y=0.8, \Delta t=0.06, \tau_{0}=1, \Delta=0.65, F_{\mathrm{u}}=2 \times$ $10^{-4}, \varepsilon=0.05$, Fig. 3 shows the dendritic pattern under a different coupling coefficient $\lambda$, it could be found that when $\lambda$ is relatively small, the main branches of grain are relatively

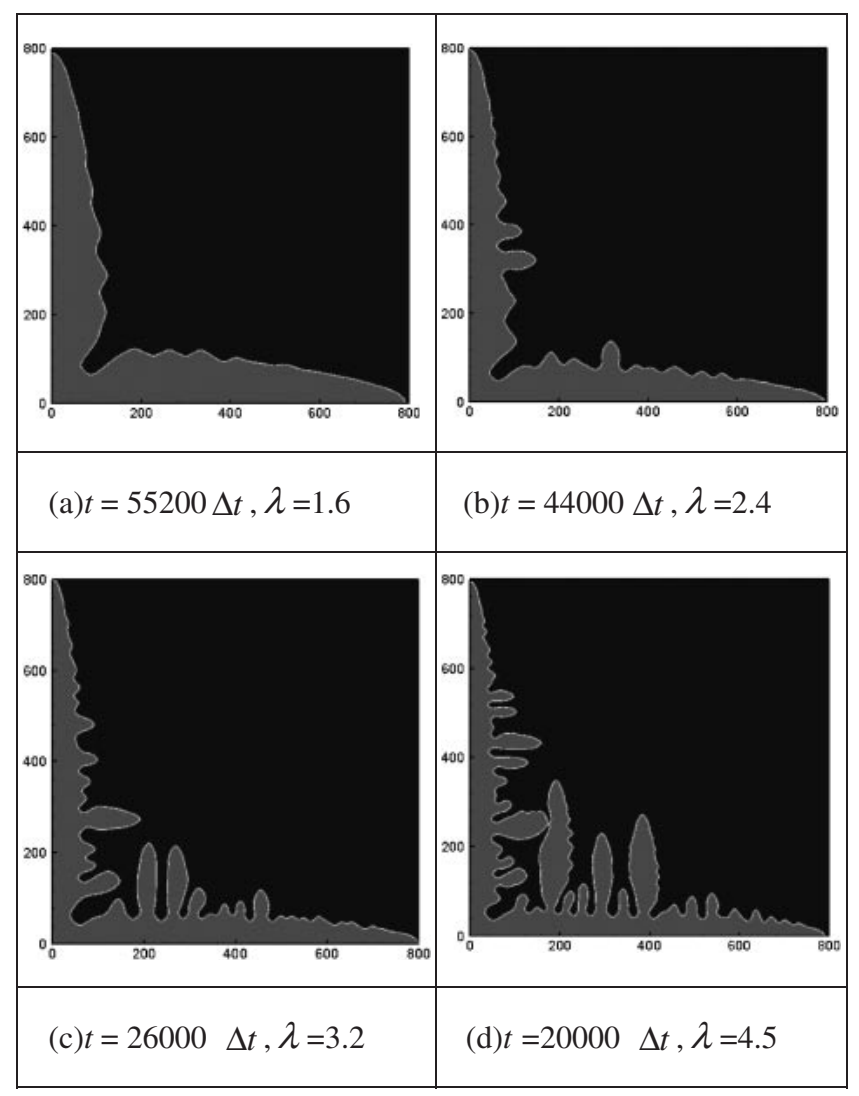

Fig. 3 The effect of the coupling coefficient $\lambda$ on side-branches.

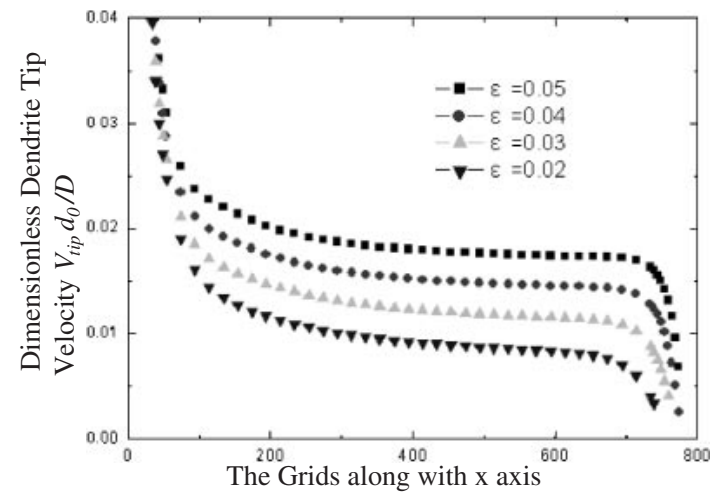

Fig. 4 The effect of the anisotropy coefficient on tip velocity.

obese, and the side-branches are very undeveloped; but with the increase of coupling coefficient $\lambda$, both the main branches and side- branches attenuate gradually, the side-branches are more and more developed. It can be explained that the bigger coupling coefficien $\lambda$ is, the more unstable the solid/liquid interface is, the easier the noise can be amplified.

\subsection{The effect of the anisotropy coefficient on the side- branches}

The anisotropy coefficient $\varepsilon$ represents the anisotropic intension of surface energy, interface thickness, and kinetics. It is not clear how to set exactly the value of the anisotropy coefficient and what its connection with real physical parameters is. However, during the simulation of microstructure evolution, the coefficient is so important to the dendritic growth that it cannot be ignored. Anisotropy coefficient $\varepsilon$ has great effects on the dimensionless dendrite tip velocity, it can be seen from the Fig. 4 that with the increment of $\varepsilon$, the dimensionless dendrite tip velocity increases accordingly, therefore, $\varepsilon$ influences the steady state behavior of the dendrite tip, which is consistent with microscopic solvability theory (MSC) conclusion about $\varepsilon$ is correlated with dendrite tip stable state behavior. ${ }^{10)}$ The operating state of the dendrite can be characterized by $\sigma^{*}=$ $\frac{2 D d_{0}}{\rho_{\mathrm{up}}^{2} V_{\text {up }}}$ and $\left.P=\frac{\rho_{\text {up }} V_{\text {up }}}{2 D} 11\right)$ where $\sigma^{*}$ is the classic stability parameter and $P$ is the dendrite tip Péclet number. It can be found that with the increase of coupling coefficient $\lambda$, the tip velocity increases accordingly, while the tip radius decreases gradually. Ivantsov theory and critical stability theory are only suitable for $\varepsilon$ 's lower situation because of neglecting $\varepsilon$ 's impact on dendrite tip. The simulation was performed by using relevant computational and material parameters as follows: $d_{0} / W_{0}=0.227, \Delta x=\Delta y=0.8, \Delta t=0.06, \tau_{0}=$ $1, \Delta=0.65$, Fig. 5 shows the dendritic pattern under different anisotropy coefficient $\varepsilon$, it could be found that when the value of $\varepsilon$ is small, the main branches and sidebranches are obese, and the DAS (Secondary Dendrite Arm Spacing) is small, with the increment of $\varepsilon$, both the main branch and side-branches become slender, and the DAS becomes small, which indicates thathas a marked effect on the side-branches, because with the increment of $\varepsilon$, the noise can be amplified quickly, the surface energy cannot restrain effectively the amplification, and then the interface becomes unstable, which causes the side-branches to split constantly. 


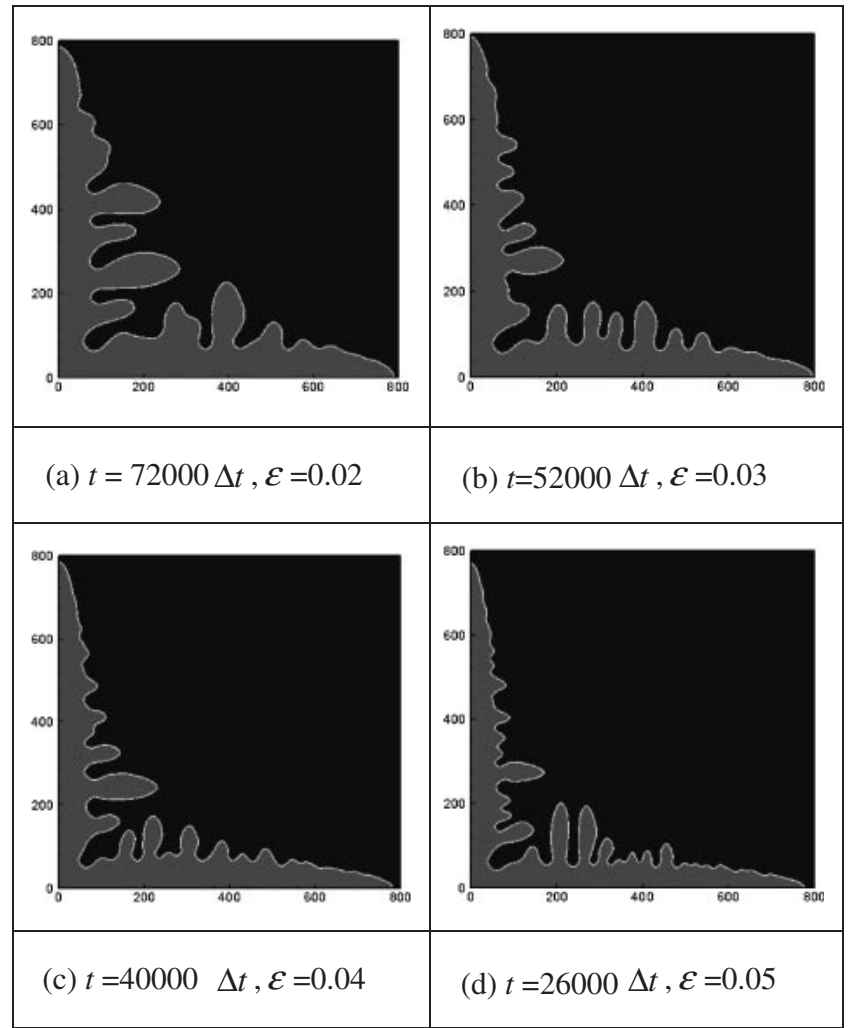

Fig. 5 The effect of anisotropy coefficient $\varepsilon$ on side-branches.

\subsection{The effect of the magnitude of thermal noise on side- braches}

The influence of thermal amplitude value on the simulation results is obvious. The simulation was performed by using relevant computational and material parameters as follows: $d_{0} / W_{0}=0.227, \tau_{0}=1, \Delta x=\Delta y=0.8, \Delta t=0.06, \varepsilon=$ 0.05 , Fig. 6(c) shows temperature field of dendrite growth at some time with noise amplitude $F_{\mathrm{u}}=10^{-4}$. From the figure, it could be found that the inclusion of thermal noise doesn't change the law of temperature field distribution, but it makes the temperature field fluctuate randomly and the highest temperature is beyond 0 , the lowest is under initial undercooling, which disagrees with the actual law of temperature field distribution. With the increase of the noise level $F_{\mathrm{u}}$, the fluctuations of the temperature field are enhanced. The curves 1 and 2 shown in Fig. 6(a) represent dendrite patterns without noise and $F_{\mathrm{u}}=10^{-4}$ at time $F_{\mathrm{u}}=25,000 \Delta t$ respectively. From the figure, we can see that durative fluctuations are formed near the interface under the condition of the noise, and side-branches appear behind the dendrite tip from several wavelengths, but the phase fields of the dendritic tip in two cases superpose. This indicates that the noise promotes the emergence of side-branches, but it doesn't influence the steady state of the dendrite tip. As shown in Fig. 6(b), the dendritic tip of pattern 1 without noise and the dendritic tip of pattern 2 with $F_{\mathrm{u}}=10^{-3}$ at time $t=25,000 \Delta t$ don't superpose, and the vertical branch velocity is greater than the horizontal branch velocity, which indicates that the greater value of noise will disturb the steady state. Therefore, the value of $F_{\mathrm{u}}$ must be tried time and again.

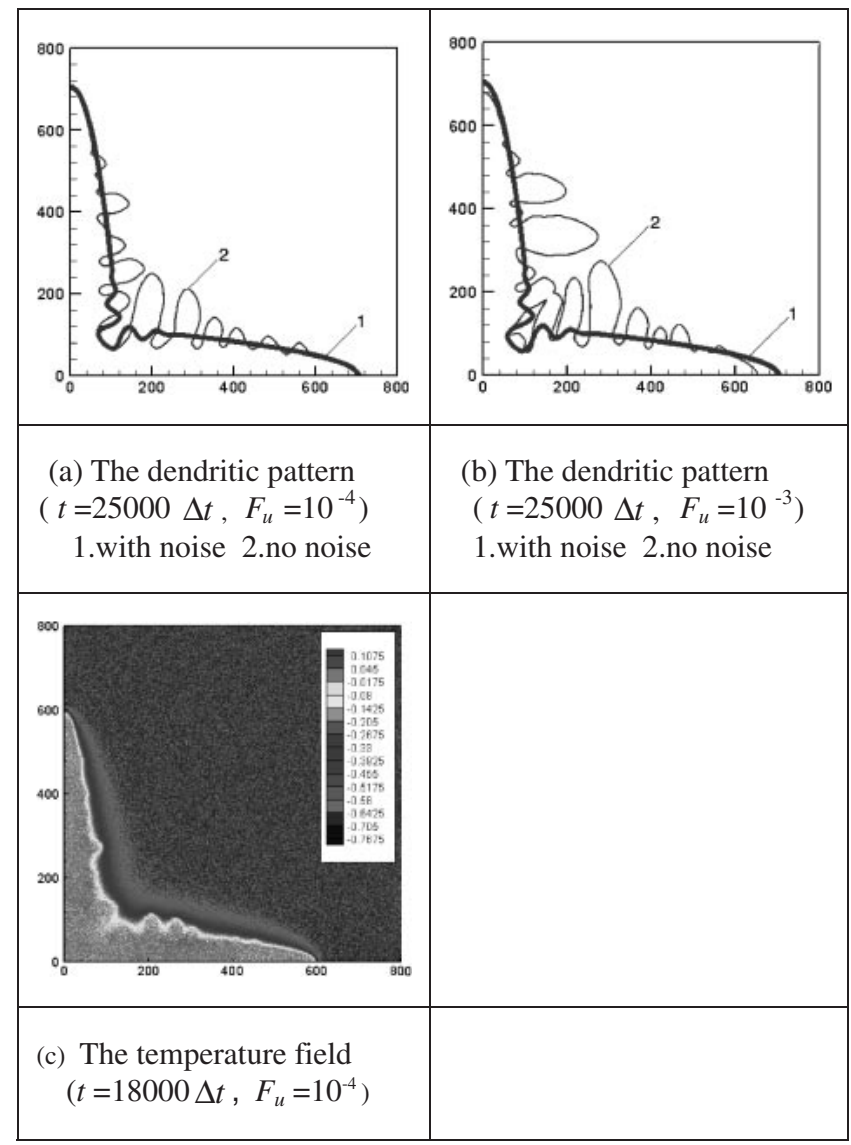

Fig. 6 The effects of the magnitude of thermal noise on side-braches.

\section{Conclusions}

(1) In the phase-field simulations of dendritic sidebranches, different phase-field parameters lead to different dendrite side-branch behaviors.

(2) With the increment of undercooling, the thermal diffusion layer around the equiaxed dendrite becomes thinner, which avails the morphology formation of the sidebranches and the equiaxed dendrite with developed sidebranches.

(3) The anisotropy coefficient $\varepsilon$ influences the steady state of the dendrite tip, the largeris, the greater the dendrite tip velocity is, the more developed the side-branches are, and the smaller the DAS is.

(4) The less the coupling coefficient $\lambda$ is, the quicker the dendrite tip velocity converges on the Green function calculation, and the more developed the side-branches are.

(5) The side-branches can not be affected by the inclusion of the nonconserved noise, so the nonconserved noise can be negligible from the point of view of computational saving for long simulation runs.

(6) The magnitude of thermal noise has an obvious influence on the temperature field. When appropriate value is assigned to $F_{\mathrm{u}}$, the noise can promote the emergence of side branches, but it does not influence the tip operating state. 


\section{Acknowledgments}

This work was supported by the National Science Foundation of China (No. 50005011 and No. 59990470).

\section{REFERENCES}

1) A. Karma and W.-J. Rappel: Phys. Rev. E 60 (1999) 3164-3624.

2) M. N. Barber, A. Barbieri and J. S. Langer: Phys. Rev. A 39 (1987) 5314-5320.

3) E. Brener and D. Temkin: Physica A 263 (1999) 338-344.
4) J. S. Langer: Phys. Rev. A 36 (1987) 3350-3358.

5) R. Kobayasi: Physica D 63 (1993) 410-423.

6) B. T. Murray, A. A. Wheeler and M. E. Glicksman: J. Cryst. Growth. 154 (1995) 386-400.

7) X. Tong, C. Beckermann, A. Karma and Q. Li: Phys. Rev. E 63 (2001) $1-16$.

8) R. Gonzálet-Cinca and L. Ramírez-Piscina: Phys. Rev. E 63 (2000) 1-9.

9) Q. Li and C. Beckermann: Acta Mater. 47 (1999) 2345-2356.

10) D. A. Kessler and H. Levine: Phys. Rev. B 33 (1986) 7867-7870.

11) A. Karma and W.-J. Rappel: Phys. Rev. E 57 (1998) 4323-4349. 